\title{
Analyzing the Symmetry Properties of a Distribution in the Focal Plane for a Focusing Element with Periodic Angle Dependence of Phase
}

\author{
Svetlana N. Khonina and Andrey V. Ustinov \\ Institution of Russian Academy of Sciences, Image Processing Systems Institute RAS, Molodogvardeiskaya Street 151, \\ Samara 443001, Russia \\ Correspondence should be addressed to Svetlana N. Khonina, khonina@smr.ru
}

Received 25 April 2012; Accepted 10 September 2012

Academic Editor: Pierre Chavel

Copyright ( $) 2012$ S. N. Khonina and A. V. Ustinov. This is an open access article distributed under the Creative Commons Attribution License, which permits unrestricted use, distribution, and reproduction in any medium, provided the original work is properly cited.

\begin{abstract}
We analyze the symmetry properties of the focal plane distribution when light is focused with an element characterized by a periodic angular dependent phase, $\sin (m \varphi)$ or $\cos (m \varphi)$. The majority of wave aberrations can be described using the said phase function. The focal distribution is analytically shown to be a real function at odd values of $m$, which provides a simple technique for generating designed wave aberrations by means of binary diffractive optical elements. Such a possibility may prove useful in tight focusing, as the presence of definite wave aberrations allows the focal spot size to be decreased. The analytical computations are illustrated by the numerical simulation, which shows that by varying the radial parameters the focal spot configuration can be varied, whereas the central part symmetry is mainly determined by the parity of $m$ : for even the symmetry order is $2 m$ and for odd is $m$.
\end{abstract}

\section{Introduction}

Various aberrations in the focusing system are known to result in a wider, distorted focal spot with disturbed axial symmetry [1]. Such an effect is normally considered to be a negative factor.

However, it has been shown [2, 3] that some types of wave aberrations enable the central focal spot size to be decreased, providing tight focusing. Note that while only primary (axisymmetric) aberrations were dealt with in [2], aberrations associated with vortex phase components on the basis of Zernike polynomials were also discussed in [3].

In Zernike polynomials, the radius dependence is polynomial and the angle dependence is trigonometric (periodic). Optical elements characterized by periodic angular changes were considered in $[4,5]$. In [4], such an element was shown to form the zero central intensity, whereas [5] also looked into diffraction-free properties of the generated light beams.
Based on the decomposition of a cosine angular dependent phase function in terms of angular harmonics, the transmission function $\exp \{i a \cos (m \varphi)\}$ was shown [5] to produce a diffraction pattern composed of $2 m$ light spots arranged on a circumference. The coaxial interference of two vortex beams with identical topological charges and opposite signs was shown to produce a similar result $[6,7]$.

At the same time, the odd-order aberrations, such as distortion and coma, have been known to appear in distribution patterns with odd symmetry $[1,8,9]$. In particular, the presence of coma $(m=3)$ results in distributions with the third-order symmetry [10], similar to the 2D Airy beams [11].

It was also shown that the product of three 1D Airy functions, rotated by the angle of $120^{\circ}$ relative to each other and characterized by the third-order symmetry, was transformed in the spectral plane into a function proportional to $\exp \left\{\operatorname{iar}^{3} \sin (3 \varphi)\right\}[12]$. 
Notice, the phase mask with sine but nonperiodic angular dependence [13] breaking symmetry can be used for selective edge enhancement.

In this paper, we analyze symmetry properties of the distribution formed in the focal plane by an optical element characterized by a periodic angular dependent phase function in the form of $\sin (m \varphi)$ or $\cos (m \varphi)$. Based on this phase relation, it is possible to describe the majority of wave aberrations, which then can be represented [14] as the decomposition in terms of Zernike functions [1].

The focal distribution is analytically shown to be described by a real function at odd values of $m$. Analytical estimates of the central part of the focal distribution are derived for an input circular aperture.

The analytical computations are illustrated by the numerical simulation, which shows that by varying the radial parameters the focal spot configuration can be varied, whereas the central part symmetry is manily determined by the even value of the angular parameter $m$.

\section{Fourier Transform of a Complex Distribution with Periodic Angular Dependence}

Assume a complex distribution with periodic angular dependence of the general form:

$$
f(r, \varphi)=\psi(r) \exp \left[-i \beta(\alpha r)^{q}\left\{\begin{array}{l}
\sin m \varphi \\
\cos m \varphi
\end{array}\right\}\right]
$$

where $\psi(r)$ is an axisymmetric function, $m$ is integer, $q$ is a positive real number, and $\alpha, \beta$ are real parameters (with $\alpha$ having the dimension of $\mathrm{mm}^{-1}$ ).

Note that various combinations of the functions $\sin (m \varphi)$ and $\cos (m \varphi)$ in the phase function of (1) can be represented as a product of functions given by (1).

The complex distribution in (1) can describe the majority of wave aberrations, which, then, can be represented [14] by the decomposition in terms of Zernike functions [1].

For the sake of specificity, choose $\sin (m \varphi)$ in (1). Note that for the $\cos (m \varphi)$ function the results will be analogous. by

The spatial Fourier spectrum for the function (1) is given

$$
\begin{aligned}
F(\rho, \theta)= & -\frac{i k \exp (i k f)}{2 \pi f} \\
& \times \int_{0}^{\infty} \psi(r)\left\{\int_{0}^{2 \pi} \exp \left[-i \beta(\alpha r)^{q} \sin m \varphi\right]\right. \\
& \left.\quad \times \exp \left[-i \frac{k}{f} r \rho \cos (\theta-\varphi)\right] d \varphi\right\} r d r
\end{aligned}
$$

where $k=2 \pi / \lambda$ is the wave number, $\lambda$ is the wavelength of laser light, $f$ is the focal length, $(r, \varphi)$ and $(\rho, \theta)$ are the polar coordinates in the input and output planes, respectively.
Let us analyze the integral over the angle in braces in (2):

$\Phi(\rho, \theta, r)$

$$
=\int_{0}^{2 \pi} \exp \left[-i \beta(\alpha r)^{q} \sin m \varphi\right] \exp \left[-i \frac{k}{f} r \rho \cos (\theta-\varphi)\right] d \varphi .
$$

The axial distribution of light $(\rho=0)$ is described by the relation independent of $m$ :

$$
\Phi(0,0 ; r)=\int_{0}^{2 \pi} \exp \left[-i \beta(\alpha r)^{q} \sin m \varphi\right] d \varphi=2 \pi J_{0}\left[\beta(\alpha r)^{q}\right] .
$$

Designating

$$
a=\beta(\alpha r)^{q}, \quad b=\frac{k r \rho}{f}
$$

and following the transformation in (3) at $m=1$

$$
\begin{aligned}
a \sin \varphi+b \cos (\varphi-\theta) & =(a+b \sin \theta) \sin \varphi+(b \cos \theta) \cos \varphi \\
& =\sqrt{a^{2}+b^{2}+2 a b \sin \theta} \cos \left(\varphi-\theta^{\prime}\right),
\end{aligned}
$$

where $\theta^{\prime}=\operatorname{arctg}[(a+b \sin \theta) / b \cos \theta]$, we obtain

$$
\begin{aligned}
& \Phi(\rho, \theta ; r) \\
& \quad=2 \pi J_{0}\left(\sqrt{\beta^{2}(\alpha r)^{2 q}+\left(\frac{k r \rho}{f}\right)^{2}+2 \beta(\alpha r)^{q}\left(\frac{k r \rho}{f}\right) \sin \theta}\right) .
\end{aligned}
$$

Replacing $\sin \varphi$ in (1) by $\cos \varphi$ (at $m=1$ ) yields an analogous relation to (7), following the relevant substitutions.

Let us consider a more general case of integer $m$. The first term in (3) can be decomposed as

$$
\begin{aligned}
\exp (-i a \sin m \varphi)= & J_{0}(a)+2 \sum_{p=1}^{\infty} J_{2 p}(a) \cos (2 p m \varphi) \\
& -2 i \sum_{p=0}^{\infty} J_{2 p+1}(a) \sin ((2 p+1) m \varphi) .
\end{aligned}
$$

Equation (8) employs the following relations [15]:

$$
\begin{aligned}
& \cos (z \sin t)=J_{0}(z)+2 \sum_{p=1}^{\infty} J_{2 p}(z) \cos (2 p t), \\
& \sin (z \sin t)=2 \sum_{p=1}^{\infty} J_{2 p+1}(z) \sin [(2 p+1) t] .
\end{aligned}
$$

To calculate the integral in (3), we can employ the wellknown relations:

$$
\begin{gathered}
\int_{0}^{2 \pi}\left\{\begin{array}{c}
\cos m \varphi \\
\sin m \varphi
\end{array}\right\} \exp [-i b \cos (\theta-\varphi)] d \varphi \\
=2 \pi(-i)^{m}\left\{\begin{array}{l}
\cos m \theta \\
\sin m \theta
\end{array}\right\} J_{m}(b) .
\end{gathered}
$$


Thus, we obtain

$$
\begin{aligned}
\Phi(\rho, \theta ; r)= & 2 \pi J_{0}(a) J_{0}(b) \\
& +4 \pi \sum_{p=1}^{\infty}(-1)^{p m} J_{2 p}(a) J_{2 p m}(b) \cos (2 p m \theta) \\
& -4 \pi i \sum_{p=0}^{\infty}(-1)^{p m}(-i)^{m} J_{2 p+1}(a) J_{(2 p+1) m}(b) \\
& \times \sin ((2 p+1) m \theta) .
\end{aligned}
$$

It should be noted that for the odd values of $m,(11)$ is a real function, because the coefficients in the third sum $i(-i)^{m}=$ $(-i)^{m-1}$ are real at odd $m$.

Taking $\cos m \varphi$ in (1), we obtain an analogous relation:

$$
\begin{aligned}
\exp (-i a \cos m \varphi)= & J_{0}(a)+2 \sum_{p=1}^{\infty}(-1)^{p} J_{2 p}(a) \cos (2 p m \varphi) \\
& -2 i \sum_{p=0}^{\infty}(-1)^{p} J_{2 p+1}(a) \cos ((2 p+1) m \varphi),
\end{aligned}
$$

which utilizes the relations [15]:

$$
\begin{aligned}
& \cos (z \cos t)=J_{0}(z)+2 \sum_{p=1}^{\infty}(-1)^{p} J_{2 p}(z) \cos (2 p t), \\
& \sin (z \cos t)=2 \sum_{p=1}^{\infty}(-1)^{p} J_{2 p+1}(z) \cos [(2 p+1) t] .
\end{aligned}
$$

Thus,

$$
\begin{aligned}
& \int_{0}^{2 \pi} \exp (-i a \cos m \varphi) \exp [-i b \cos (\theta-\varphi)] d \varphi \\
& =2 \pi J_{0}(a) J_{0}(b)+4 \pi \sum_{p=1}^{\infty}(-1)^{p(m+1)} J_{2 p}(a) J_{2 p m}(b) \cos (2 p m \theta) \\
& -4 \pi i \sum_{p=0}^{\infty}(-1)^{p(m+1)}(-i)^{m} J_{2 p+1}(a) J_{(2 p+1) m}(b) \\
& \quad \times \cos ((2 p+1) m \theta) .
\end{aligned}
$$

For the odd values of $m$, the function in (14) is real.
Substitute (11) in (2):

$$
\begin{aligned}
& F(\rho, \theta)=-\frac{i k}{f} \exp (i k f) \\
& \times \int_{0}^{\infty} \psi(r)\left\{J_{0}\left[\beta(\alpha r)^{q}\right] J_{0}\left(\frac{k}{f} r \rho\right)\right. \\
& \quad-2 i(-i)^{m} J_{1}\left[\beta(\alpha r)^{q}\right] J_{m} \\
& \quad \times\left(\frac{k}{f} r \rho\right) \sin (m \theta) \\
&+2(-1)^{m} J_{2}\left[\beta(\alpha r)^{q}\right] J_{2 m} \\
&\left.\quad \times\left(\frac{k}{f} r \rho\right) \cos (2 m \theta)-\cdots\right\} r d r .
\end{aligned}
$$

Equation (15) can be rearranged as follows:

$$
\begin{array}{r}
F(\rho, \theta)=-\frac{i k}{f} \exp (i k f)\left\{\Psi_{0}(\rho)-2(-i)^{m-1} \Psi_{1}(\rho) \sin (m \theta)\right. \\
\left.+2(-1)^{m} \Psi_{2}(\rho) \cos (2 m \theta)-\cdots\right\}
\end{array}
$$

where

$$
\Psi_{p}(\rho)=\int_{0}^{\infty} \psi(r) J_{p}\left[\beta(\alpha r)^{q}\right] J_{p m}\left(\frac{k}{f} r \rho\right) r d r .
$$

Let us obtain approximate estimates of the integrals in (17), putting

$$
\psi(r)= \begin{cases}1, & r \leq R \\ 0, & r>R\end{cases}
$$

and assuming $J_{0}(z) \approx 1-z^{2} / 4, J_{n}(z) \approx(z / 2)^{n} / n !$.

Then,

$$
\begin{aligned}
\Psi_{0}(\rho) & =\int_{0}^{R} J_{0}\left[\beta(\alpha r)^{q}\right] J_{0}\left(\frac{k}{f} r \rho\right) r d r \\
& \approx \int_{0}^{R}\left[1-\frac{\beta^{2}(\alpha r)^{2 q}}{4}-\frac{(k r \rho)^{2}}{4 f^{2}}\right] r d r \\
& =\frac{R^{2}}{2}-\frac{(\beta R)^{2}(\alpha R)^{2 q}}{8(q+1)}-\left(\frac{k \rho R^{2}}{4 f}\right)^{2}, \\
\Psi_{p}(\rho) & =\int_{0}^{R} J_{p}\left[\beta(\alpha r)^{q}\right] J_{p m}\left(\frac{k}{f} r \rho\right) r d r \\
& \approx \frac{1}{p !(p m) !} \int_{0}^{R}\left[\frac{\beta(\alpha r)^{q}}{2}\right]^{p}\left(\frac{k}{2 f} r \rho\right)^{p m} r d r \\
& =\frac{\beta^{p} \alpha^{p q}(k \rho)^{p m}}{p !(p m) ! 2^{p}(2 f)^{p m}} \cdot \frac{R^{p(q+m)+2}}{p(q+m)+2}, \quad p>0 .
\end{aligned}
$$


TABLE 1: Simulated focusing results for simple functions $\psi(r)$ and different parameters.

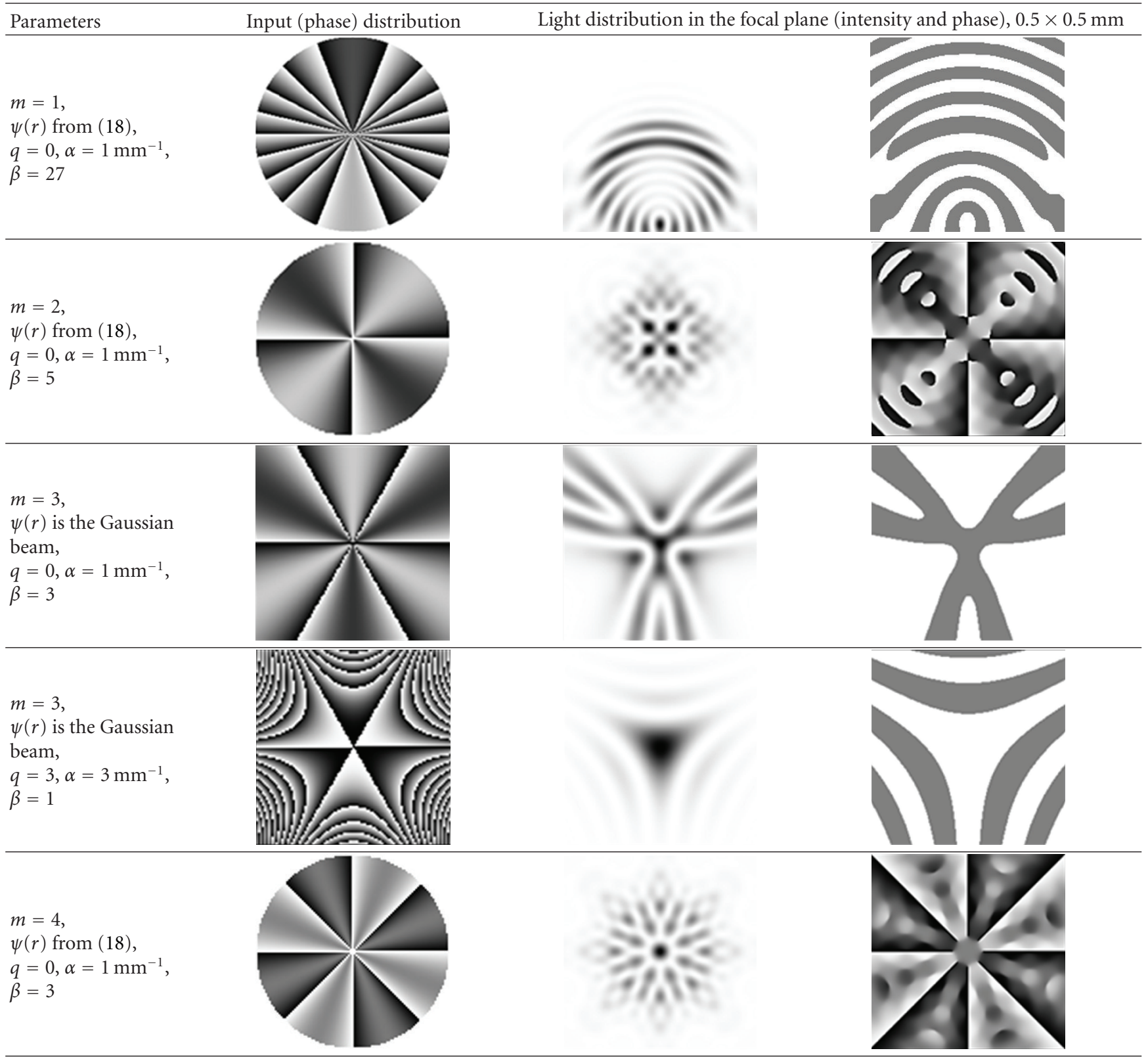

It can be seen from (19a) and (19b) that the major contribution to the central part of the focal plane $($ at $\rho \rightarrow 0)$ is provided by the terms with lower-order $p$. Truncating (16) at the first two terms, the intensity in the central part of the focal plane can be estimated for the even values of $m$ as

$$
|F(\rho, \theta)|^{2}=\left(\frac{k}{f}\right)^{2}\left[\Psi_{0}^{2}(\rho)+4 \Psi_{1}^{2}(\rho) \sin ^{2}(m \theta)\right],
$$

and for odd $m$ as

$$
\begin{aligned}
|F(\rho, \theta)|^{2}=\left(\frac{k}{f}\right)^{2}[ & \Psi_{0}^{2}(\rho)+4 \Psi_{1}^{2}(\rho) \sin ^{2}(m \theta) \\
& \left.-4(-1)^{(m-1) / 2} \Psi_{0}(\rho) \Psi_{1}(\rho) \sin (m \theta)\right],
\end{aligned}
$$

with the first term being independent of the angle and the second term resulting in $2 m$ uniformly distributed intensity peaks of equal height (at $\sin (m \theta)= \pm 1$ ). The third term is only present for odd $m$, introducing corrections into the peaks: depending on the sign of the term containing $\sin (m \theta)$, half of the peaks are enhanced, while the other half are suppressed, which results in $m$ clearly expressed maximums.

Thus, (20a) and (20b) suggest that the symmetry of the central part of the focal distribution is determined by the parity of $m$ : for even $m$, the symmetry order is $2 m$, for odd $m$, the symmetry order is $m$.

By varying the parameters $\beta, \alpha, q$, and $m$, the values of the coefficients in (20a) and (20b) can be varied, thus changing the symmetry of the intensity pattern in the focus. 
TABLE 2: Simulation results for different parameters and the form of the $\psi(r, \varphi)$ function, $q=0$.

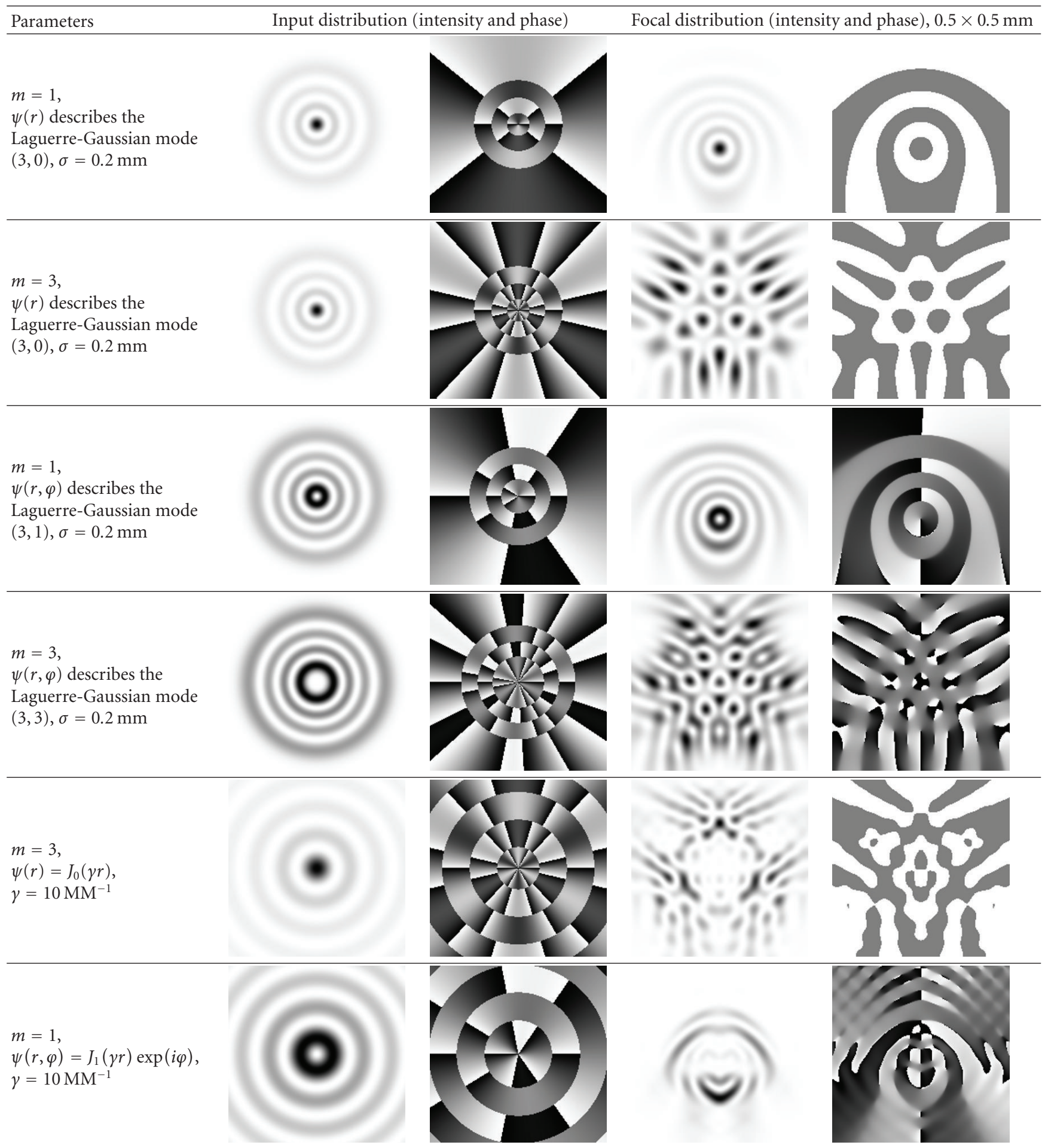

\section{Numerical Simulation}

The numerical simulation based on (2) was conducted for the following parameters: wavelength of incident light, $\lambda=$ $633 \mathrm{~nm}$, focal length, $f=100 \mathrm{~mm}$.

Table 1 gives the simulation results based on (1), for $\sin (m \varphi)$, for different values of $m$ and different parameter's values of $\alpha, \beta$, and $q$, with the $\psi(r)$ function being given by (18), describing a circular diaphragm of radius $R=$ $1 \mathrm{~mm}$, and a Gaussian beam $\exp \left(-r^{2} / \sigma^{2}\right)$ of waist radius $\sigma=0.5 \mathrm{~mm}$.

Notice that the input phase distributions (shown in the second column of the table) have the same values in opposite points on diameter line for even $m$ and negative values for 
odd $m$. It is easy to confirm by $\sin (2(x+\pi))=\sin (2 x)$, $\sin (x+\pi)=-\sin (x)$. Both of cases differ from a situation with a spiral phase $\exp (\operatorname{im} \varphi)$ where a phase difference at a symmetric position is $m \pi$ [13], so the spiral phase is used for the radial Hilbert transform of $m$ th order. In our case a phase difference in symmetric points can explain focal distribution. Since at odd $m$ in focus there are identical phases with opposite sign the field will be real valued $(\exp (i \psi)+\exp (-i \psi) \sim \cos (\psi))$. For even $m$, identical values are summarized, therefore the phase structure is regenerated in focal domain.

Table 2 presents the focusing results for $q=0$ and $\beta=5$, with the values of $m$ and $\psi(r)$ being varied.

The effect of replacing the axisymmetric function $\psi(r)$ with a more general function $\psi(r, \varphi)$ with a harmonic angle dependence, $\exp (i l \varphi)$, was also studied.

The four top lines are for the function $\psi(r, \varphi)$ described by the Fourier-transform-invariant Laguerre-Gaussian modes [15] of different orders $(n, l)$, including those with a vortex function $\exp (i l \varphi)$. It is noteworthy that the vortex angle component does not exert such an essential influence on the focal pattern structure as the periodic function in question $\sin (m \varphi)$ does. The comparison of lines 1 and 3 shows that a phase vortex introduced in the input plane results in a similar pattern in the focal plane, without changing the major distribution structure (except for the zero intensity in the singular point).

When $m=1$, the resulting distribution is similar to that associated with the coma-type aberration introduced in the focusing system; when $m=3$, the distribution is described by the product of three 1D Airy functions, rotated relative to each other [12]. Note, however, that in the latter case the product is actually composed of somewhat different functions.

In two bottom lines of Table 2, the function $\psi(r, \varphi)$ is given by the Bessel modes $J_{l}(\gamma r) \exp (i l \varphi)$, which produce a Fourier spectrum in the form of a narrow ring of radius proportional to $\gamma$. The simulation results show that the annular focal structure produced by the Bessel modes undergoes essential variations caused by the periodic angle change.

\section{Conclusions}

We have analyzed symmetry properties of the light distribution generated in the focal plane by focusing the light beam with a periodic angular dependent phase given by $\sin (m \varphi)$ or $\cos (m \varphi)$.

The focal distribution of light is analytically shown to be a real function when the value of $m$ is odd. Thus, in a way similar to [12], phase distributions associated with the corresponding specified aberration types can be formed in the focal plane of a conventional spherical lens with the aid of binary diffractive optical elements. Such a possibility may prove useful in tight focusing, where the presence of certain wave aberrations enables the focal spot size to be reduced $[2,3]$. In particular, for a circularly polarized beam the spot size can be reduced by the presence of coma $(m=$ 3 ), corresponding to the $2 \mathrm{D}$ superpositions of Airy beams $[11,12]$.
The analytical computations have been illustrated by the numerical simulation, which has shown that by varying the radial parameters, the focal distribution configuration can be varied; meanwhile, the central part symmetry is mainly determined by the parity of the angle parameter $m$ : when $m$ is even, the central part has the $2 m$-order symmetry, and when $m$ is odd-the symmetry is $m$ order. Note that although the introduced vortex component $\exp (i l \varphi)$ is preserved in the focal plane, it does not have an essential effect on the focal distribution structure when compared with the periodic function in question $\sin (m \varphi)$.

\section{Acknowledgments}

The work was financially supported by the RFBR Grants no. 10-07-00109-a, 10-07-00438-a, and the RF Presidential Grant for Support of Leading Scientific schools NSh4128.2012.9.

\section{References}

[1] M. Born and E. Wolf, Principles of Optics, Pergamon Press, Oxford, UK, 1968.

[2] R. Kant, "Superresolution and increased depth of focus: an inverse problem of vector diffraction," Journal of Modern Optics, vol. 47, no. 5, pp. 905-916, 2000.

[3] S. N. Khonina, A. V. Ustinov, and E. A. Pelevina, "Analysis of wave aberration influence on reducing focal spot size in a high-aperture focusing system," Journal of Optics, vol. 13, no. 9, Article ID 095702, 13 pages, 2011.

[4] J. Ojeda-Castañeda, P. Andrés, and M. Martínez-Corral, "Zero axial irradiance by annular screens with angular variation," Applied Optics, vol. 31, no. 22, pp. 4600-4602, 1992.

[5] S. Topuzoski and L. Janicijevic, "Diffraction characteristics of optical elements designed as phase layers with cosine-profiled periodicity in azimuthal direction," Journal of the Optical Society of America A, vol. 28, no. 12, pp. 2465-2472, 2011.

[6] S. N. Khonina, V. V. Kotlyar, V. A. Soifer, K. Jefimovs, and J. Turunen, "Generation and selection of laser beams represented by a superposition of two angular harmonics," Journal of Modern Optics, vol. 51, no. 5, pp. 761-773, 2004.

[7] V. V. Kotlyar, S. N. Khonina, R. V. Skidanov, and V. A. Soifer, "Rotation of laser beams with zero of the orbital angular momentum," Optics Communications, vol. 274, no. 1, pp. 814, 2007.

[8] G. D. Love, "Wave-front correction and production of Zernike modes with a liquid-crystal spatial light modulator," Applied Optics, vol. 36, no. 7, pp. 1517-1524, 1997.

[9] B. R. Boruah and M. A. A. Neil, "Susceptibility to and correction of azimuthal aberrations in singular light beams," Optics Express, vol. 14, no. 22, pp. 10377-10385, 2006.

[10] A. B. Budgor, "Exact solutions in the scalar diffraction theory of aberrations," Applied Optics, vol. 19, no. 10, pp. 1597-1600, 1980.

[11] S. N. Khonina, "Specular and vortical Airy beams," Optics Communications, vol. 284, no. 19, pp. 4263-4271, 2011.

[12] E. Abramochkin and E. Razueva, "Product of three Airy beams," Optics Letter, vol. 36, no. 19, pp. 3732-3734, 2011.

[13] M. K. Sharma, J. Joseph, and P. Senthilkumaran, "Selective edge enhancement using anisotropic vortex filter," Applied Optics, vol. 50, no. 27, pp. 5279-5286, 2011. 
[14] S. N. Khonina, V. V. Kotlyar, and Y. Wang, "Diffractive optical element matched with Zernike basis," Pattern Recognition and Image Analysis, vol. 11, no. 2, pp. 442-445, 2001.

[15] M. Abramowitz and I. A. Stegun, Eds., Handbook of Mathematical Functions: With Formulas, Graphs, and Mathematical Tablesby, Courier Dover Publications, 1964. 

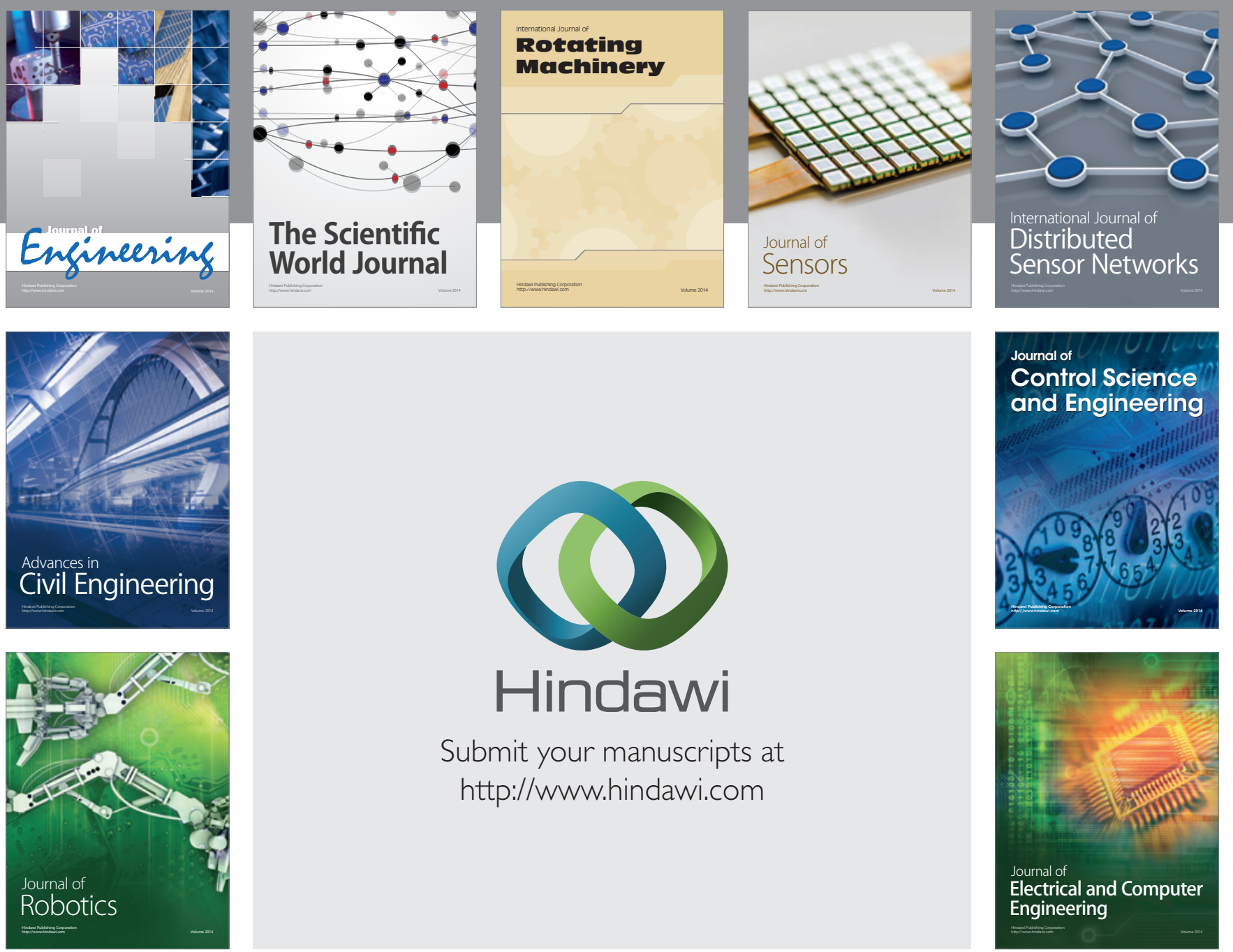

Submit your manuscripts at

http://www.hindawi.com
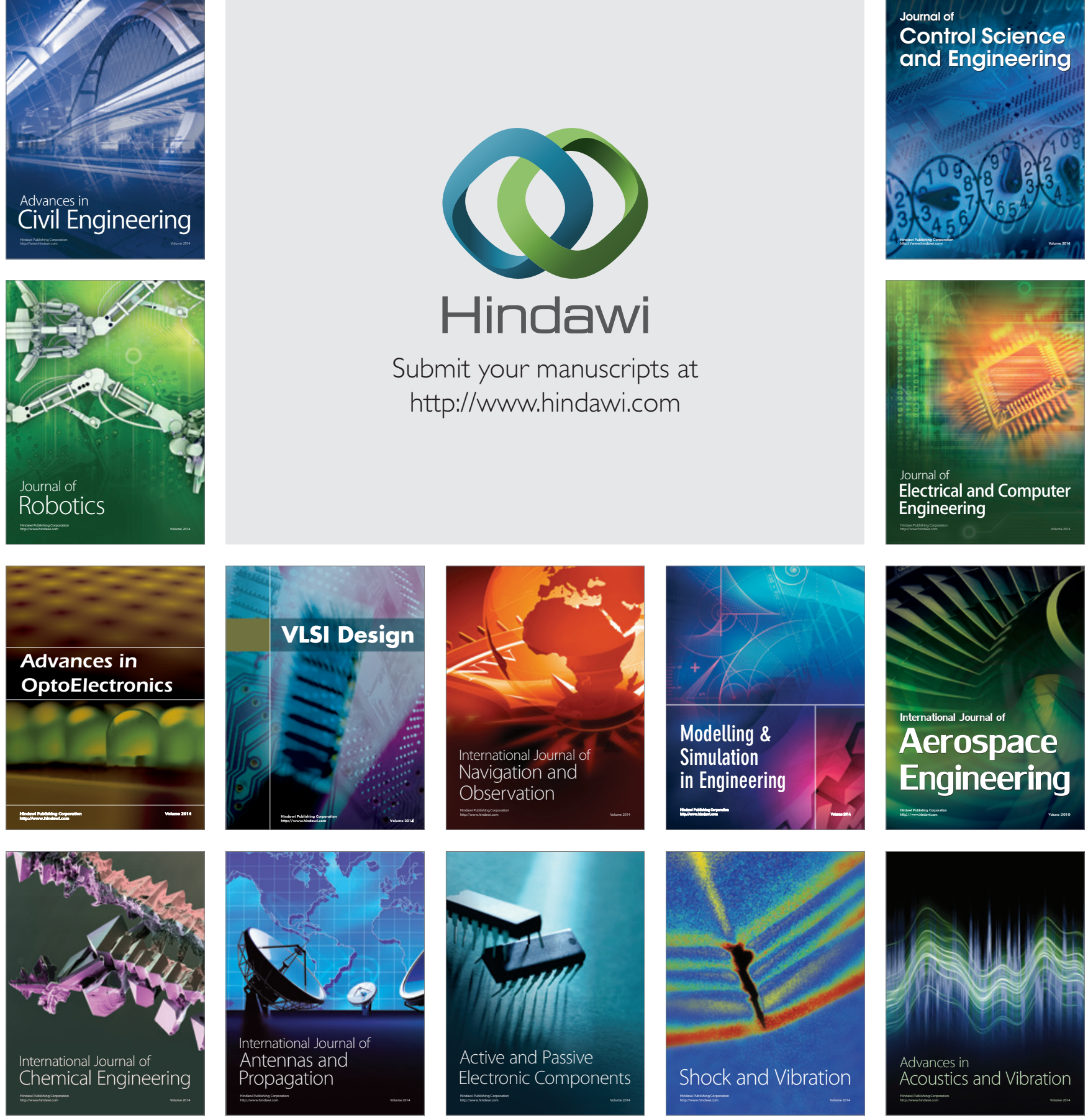